\title{
Apnea como forma de presentación de una infección por metapneumovirus humano
}

\author{
Leonor Jofré M., Vivian Luchsinger F., Guillermo Zepeda F., Antonio Rojas C. y Andrés Muñoz A.
}

\section{Apnea as a presenting symptom in human metapneumovirus infection}

Human metapneumovirus (hMPV), a recently described pathogen of lower respiratory tract infections (LRTI), has been detected in 5,4\% of Chilean infants hospitalized for LRTI whom are negative for adenovirus, respiratory syncytial virus, influenza and parainfluenza viruses. hMPV may cause bronchiolitis or pneumonia in hospitalized patients, and ocassionally require admission to intensive care units and mechanical ventilation. The infection has been associated with apnea, especially in preterm infants. Nosocomial dissemination has also been described. We present the case of a one-month-of age premature infant with apnea, and infection caused by hMPV of probable nosocomial aquisition. Clinical features of hMPV infection are reviewed and its association with apnea and nosocomial transmission is discussed. hMPV should be included in the routine diagnosis of respiratory viruses in infants with apnea and should be considered among the respiratory pathogens associated with nosocomial transmission.

Key words: apnea; respiratory tract infections; human metapneumovirus; hMPV; infection control; nosocomial infection.

Palabras claves: apnea; infección respiratoria; metapneumovirus humano; MPVh; control de infecciones; infección intrahospitalaria.

\section{Introducción}

$\mathbf{L}$ as infecciones respiratorias agudas bajas (IRAB) son un importante problema de salud pública en Chile; constituyendo la principal causa de hospitalización bajo dos años de edad.

Setenta a $90 \%$ de las IRAB son producidas por agentes virales, entre los que destacan virus respiratorio sincicial (VRS), adenovirus (ADV) y virus influenza $(\text { Flu })^{1,2}$, además de rhinovirus, coronavirus, parainfluenza (paraFlu) y el recientemente descrito metapneumovirus humano (MPVh) ${ }^{3}$. El agente más frecuente es VRS, detectado en 39 a $70 \%$ de los niños con IRAB en época de brote ${ }^{4}$, seguido por ADV en 2 a $24 \% \%^{5}$ y Flu en $8 \%$ de los pacientes ${ }^{6}$. En una experiencia chilena, se demostró la presencia de MPVh en 5,4\% de los lactantes hospitalizados por IRAB, con estudio para VRS, ADV, Flu y paraFlu negativo ${ }^{7}$.

Metapneumovirus humano pertenece a la familia de los Paramyxoviridae, subfamilia Pneumovirinae, género Metapneumovirus. Al igual que el VRS, posee ARN y manto. Se han descrito dos subgrupos, denominados A y B, subdivididos en dos grupos a su vez: $\mathrm{A} 1, \mathrm{~A} 2, \mathrm{~B} 1$ y $\mathrm{B} 2$, que pueden circular en una misma temporada ${ }^{9,10}$.

Este virus se asocia a cuadros de obstrucción y neumonía, entre otras manifestaciones, y su espectro clínico aún no se encuentra totalmente definido. Presentamos el caso de un lactante que ingresó por apnea y en el cual se detectó la presencia de MPVh.

\section{Caso clínico}

Lactante de sexo masculino, nacido con 35 semanas de gestación, adecuado para la edad gestacional, peso de nacimiento de $2.660 \mathrm{~g}$, Apgar 9-10, sin patologías en el período de $\mathrm{RN}$ inmediato y alimentado con lactancia materna exclusiva.

Al mes de vida (noviembre de 2005), consultó por un cuadro de tos de un día de evolución, decaimiento, inapetencia y vómitos post-prandiales. En el trayecto al hospital presentó un episodio de apnea, que respondió al estímulo táctil realizado por los padres. Ingresó respirando en forma espontánea, pero con pausas respiratorias a repetición, lo que motivó su traslado a la unidad de cuidados intensivos pediátricos (UCIP).

Al ingreso se constató un niño en regulares condiciones generales, reactivo, conectado, con estabilidad hemodinámica, y estertores aislados a la auscultación pulmonar. Debido a un nuevo episodio de apnea, de 40
Universidad de Chile,

Santiago, Chile

Hospital Clínico

Servicio de Pediatría (UM, GZF,

ARC, AMA)

Facultad de Medicina

Instituto de Ciencias Biomédicas

Programa de Virología (VLF)

Recibido: 10 de septiembre de 2006 Aceptado: 21 de marzo de 2007

Correspondencia a:

Leonor Jofré Morales

leonorjofre@gmail.com 
segundos de duración, debió ser conectado a ventilación mecánica invasora (VMI).

Entre los exámenes realizados al ingreso, presentó una glicemia normal, hematocrito de $37 \%$, hemoglobina $12,4 \mathrm{~g} / \mathrm{dl}$, leucocitos de 6.470 céls $/ \mathrm{mm}^{3}$, (segmentados $29 \%$, baciliformes $0 \%$, linfocitos $59 \%$ ) y plaquetas 245.000 céls $/ \mathrm{mm}^{3}$ y PCR 0,6 mg/L. El LCR fue normal, el urocultivo y los hemocultivos negativos. La ecografía encefálica no mostró alteraciones. En la radiografía de tórax de ingreso se evidenció un infiltrado intersticio alveolar bilateral, sin imágenes de consolidación ni de efusión (Figura 1). El estudio de ADV por aislamiento viral e IFI, de VRS, Flu y paraFlu por IFI, y de Bordetella pertussis por IFD fue negativo.

Se inició tratamiento empírico con cefotaxima y ampicilina. Evolucionó con aumento de los parámetros ventilatorios y necesidad de $\mathrm{O}_{2}$ con $\mathrm{FiO}_{2}$ de $100 \%$, los gases se mantuvieron con tendencia a la acidosis respiratoria, lo que dificultó el manejo del paciente, pasando por varias modalidades en el ventilador convencional, y un importante componente obstructivo, por lo que se agregó metilprednisolona. Por la gravedad del paciente y ante la sospecha de un cuadro de etiología viral, se solicitó un estudio viral complementario y amplio, que incluyó la pesquisa de MPVh. En el aspirado traqueal (AT) obtenido al séptimo día de hospitalización, se buscó CMV por técnica de aislamiento viral rápido (shell vial), $\mathrm{ADV}$ por técnica de cultivo celular e IFI, VRS por técnica de IFI, Chlamydia trachomatis y Pneumocystis jiroveci mediante RPC y cultivo bacteriano cuantitativo de aspirado traqueal, todos los cuales fueron negativos. Desde la secreción bronquial obtenida, se amplificó un fragmento del gen $\mathrm{N}$ de MPVh mediante la RPC en tiempo real (RPC-LC) $)^{10}$ (Figura 2).

Al octavo día de evolución experimentó un deterioro, con bradicardia e hipotensión arterial, que respondió al aporte de volumen. En una nueva radiografía de tórax se observó un compromiso alveolar multifocal, hiperinsuflación bilateral, sin evidencia de efusión (Figura 3) y una ecocardiografía que no mostró alteraciones. Se decidió agregar eritromicina al tratamiento inicial cambiándose, posteriormente, a azitromicina.

A los 10 días de evolución, tras completar 10 días de cefotaxima y cinco de azitromicina, se logró extubar, no presentando pausas respiratorias ni apneas durante dos días. El aporte de $\mathrm{O}_{2}$ se suspendió al tercer día post extubación, manteniéndose en buenas condiciones generales y sin dificultad respiratoria. La radiografía de tórax de control mostró imágenes intersticiales bilaterales, algo más confluentes en la zona paracardíaca izquierda. Se dio de alta con kinesioterapia ambulatoria, por persistencia de la signología húmeda.

Por la reaparición de cianosis durante una sesión de kinesioterapia ambulatoria y la presencia de atelectasia del lóbulo superior izquierdo e infiltrado intersticial bilateral, debió ser rehospitalizado. En esta nueva oportunidad, la IFI para ADV, VRS, Flu y paraFlu fueron negativas. Tras un período de observación, fue nuevamente dado de alta. Continuó presentando pausas respiratorias por lo que se efectuó un estudio polisomnográfico y metabólico con resultados normales. Para descartar fibrosis quística, se le solicitó estudio genético que fue normal. En los controles ambulatorios se detectó hiperreactividad bronquial, tratándose con corticosteroides inhalatorios. El desarrollo pondoestatural y psicomotor es actualmente normal para su edad.

\section{Discusión}

Metapneumovirus es un agente de infección respiratoria en circulación desde hace 50 años, aunque descrito recién en el año 2001, por un grupo de investigadores holandeses ${ }^{3}$. Presenta una distribución universal, habiéndose detectado en E.U.A y América del Norte $^{11-15}$, Europa ${ }^{16-20}$, Australia ${ }^{21}$, Asia $^{22-23}$ África ${ }^{24,25}$ y Latinoamérica ${ }^{7,26-28}$. Se describen brotes por este agente a fines del invierno y comienzos de la primavera, los que se prolongan hasta después de finalizar el brote de VRS $^{29}$. Nuestro caso fue hospitalizado en noviembre del año 2005, fecha en que no había circulación de VRS en Santiago, Chile.

MPVh produce manifestaciones clínicas similares al VRS y afecta en forma más frecuente a los lactantes bajo un año de edad ${ }^{30}$; ya a los cinco años, prácticamente todos los niños tienen anticuerpos contra este agente $^{3}$. La inmunidad no es duradera, por lo que puede haber re-infecciones posteriores ${ }^{31}$. Puede ocasionar entre 2 y $18 \%$ de las IRAB que se hospitalizan ${ }^{32-35}$ y $12 \%$ de las consultas ambulatorias ${ }^{29}$. Síntomas acompañantes descritos son: tos (en 90\% de los casos), rinorrea $(75 \%)$, signología obstructiva $(57 \%)$, fiebre (50\%), vómitos (41\%), irritabilidad (37\%), OMA (33\%), disfagia $(31 \%)$, laringitis obstructiva $(8-14 \%)$, diarrea (6-35\%), exantema $(11 \%)$, y conjuntivitis $(3-18 \%)^{36-41}$. Quienes requieren hospitalización presentan una mayor frecuencia de bronquiolitis, neumonía, apnea, necesidad de conexión a VMI e ingreso a una UCIP ${ }^{13,42-50}$.

La prueba de elección para el diagnóstico de MPVh es la detección del genoma viral mediante transcripción reversa y RPC, la que presenta una alta sensibilidad y especificidad ${ }^{51}$. El aislamiento viral tiene bajo rendimiento y requiere de personal e infraestructura apropiados; aún no se dispone de métodos eficaces para detectar antígenos ${ }^{7}$. No se ha definido la muestra más apropiada para investigar este agente en un pa- 
ciente con neumonía; si bien, en la mayoría de los casos se han estudiado aspirados nasofaríngeos, en este paciente se detectó en el AT.

Aunque la detección de MPVh en el caso presentado se realizó al séptimo día de hospitalización, planteamos su participación en el cuadro clínico, considerando la ausencia de los virus respiratorios circulantes clásicos -como VRS- en dicho período y de otros niños internados con patología respiratoria en la UCIP, la hospitalización del paciente en régimen de aislamiento individual y el estudio negativo para ADV y VRS. Más aún, la época del año en que ocurrió, lejos de la "temporada de VRS", también apoya al diagnóstico de MPVh.

La presentación de apneas durante el curso de una infección por MPVh ha sido descrita en 2 a $6 \%$ de los casos en algunas series, aumentando a $40 \%$ en el caso de prematuros, en quienes se describe el llamado complejo apnea-bradicardia ${ }^{52-54}$. La causa de esta forma de presentación aún no está establecida y pareciera ser de tipo obstructivo. En infecciones por VRS se ha encontrado asociación con apnea en 16 a $20 \%$ de los casos, con un mayor riesgo en lactantes bajo dos meses y en prematuros ${ }^{55}$. En el caso de VRS, los determinantes parecieran ser varios: gravedad del cuadro que ocasiona algún grado de hipoxemia; compromiso extenso del parénquima pulmonar; alteración de la sensibilidad de los receptores laríngeos, que al ser estimulados inducen el episodio de apnea y la inmadurez del centro de control respiratorio ${ }^{56,57}$. Las semejanzas entre VRS y el MPVh permiten postular la participación de algunos de estos factores como causa de la apnea en pacientes con antecedentes de prematurez e inmadurez del sistema respiratorio, como sería el caso descrito. Hasta ahora no se ha demostrado un efecto directo del MPVh en el centro respiratorio. En este paciente se descartaron causas de apnea de tipo central con la polisomnografía y el estudio metabólico.

Metapneumovirus puede ser diseminado en el ambiente nosocomial ${ }^{57}$, situación que se descartaría en este caso por su permanencia en una unidad de aislamiento individual y la ausencia de otros pacientes con cuadros respiratorios en ese período en la UCIP. Sin embargo, considerando un período de incubación que se supone entre cinco y seis días ${ }^{12,46}$ y una excreción viral que duraría entre una y cuatro semanas, es posible que esta fuera la vía de infección de un lactante de ocho meses hospitalizado por una enfermedad metabólica durante un largo tiempo en la unidad. En el aspirado nasofaríngeo obtenido al segundo día de inicio de síntomas respiratorios, se amplificó un fragmento del genoma de MPVh. Después de este paciente no se presentaron nuevos casos en la UCIP. La vía

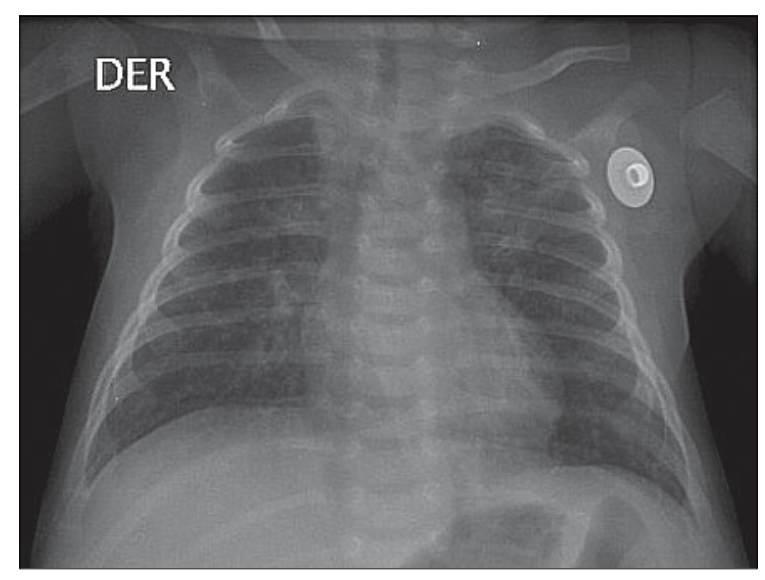

Figura 1. Radiografía de tórax de ingreso con compromiso intersticio-alveolar bilateral.

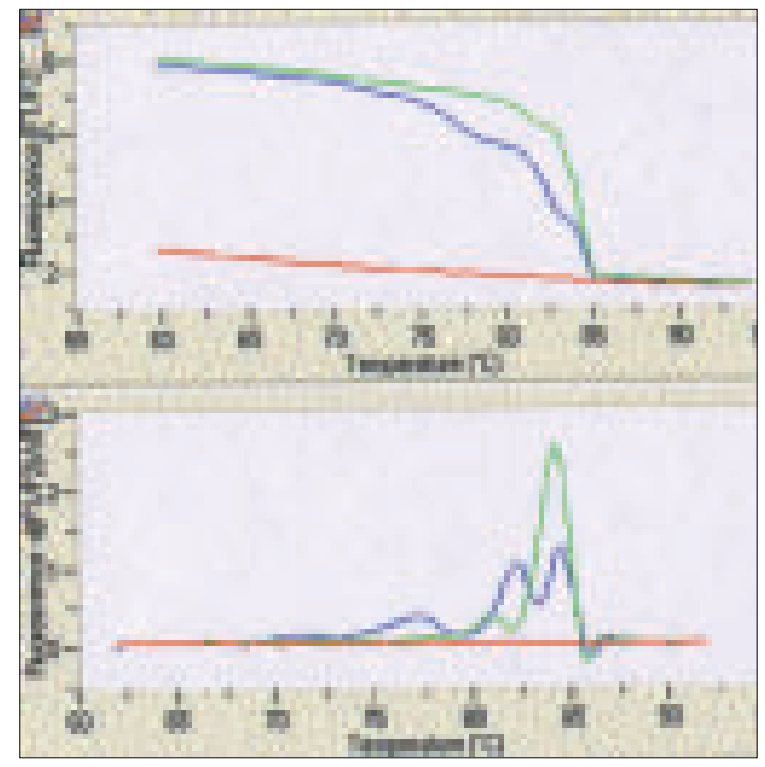

Figura 2. Curva de fluorescencia y de temperatura de alineamiento (melting) de la RPC-LC del gen N de MPVh. Curva roja: control negativo; Curva verde: control positivo; Curva azul: muestra del paciente.

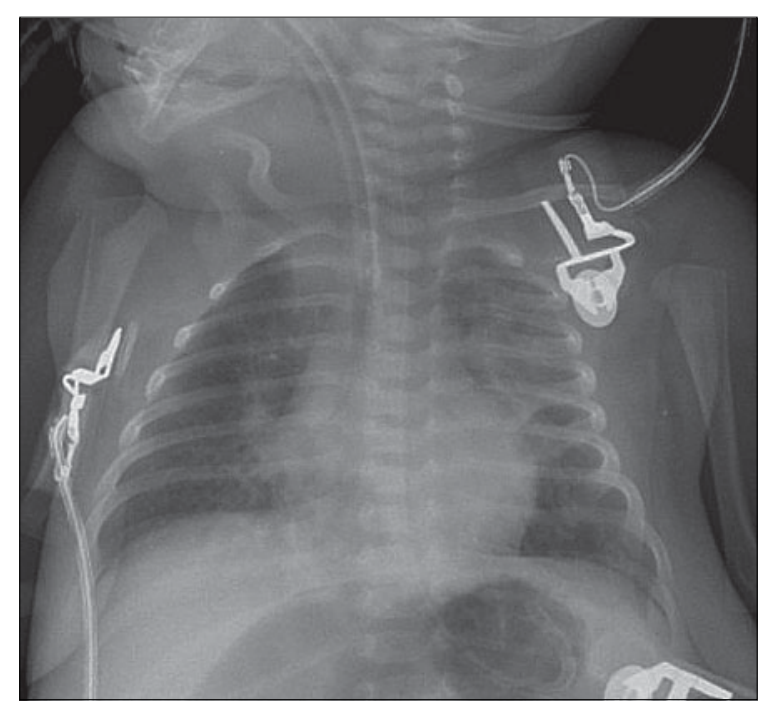

Figura 3. Radiografía de tórax durante evolución con compromiso multifocal e hiperinsuflación bilateral. 
de transmisión sería similar a la del VRS, a través de gotitas y por contacto directo con secreciones.

Se ha descrito una evolución más grave en pacientes con bronquiolitis co-infectados con VRS, los que debieron ser conectados a VMI en una UCIP ${ }^{58-59}$. Esto también ocurriría en pacientes inmunocomprometidos, población donde la infección puede llegar a ser fatal ${ }^{60}$, en pacientes con patología respiratoria crónica $^{52}$ y en adultos sobre 65 años de edad ${ }^{35}$. La hiper-reactividad bronquial, como la presentada en este caso, se ha asociado a MPVh, detectándose con mayor frecuencia este agente en pacientes con exacerbación de un cuadro asmático y en lactantes con obstrucción ${ }^{12,37,61-62}$.

Del caso descrito, concluimos la necesidad de complementar el estudio etiológico, buscando nuevos agentes respiratorios virales como MPVh, en aquellos pacientes con cuadros respiratorios bajos, que tengan un estudio negativo para los agentes respiratorios tradicionales, considerando, que su evolución puede ser grave, como ocurrió en este paciente. Si bien la edad de presentación no es la más frecuente, esta posibilidad debe ser barajada en casos de apnea y prematurez, que coincidan con la época de circulación del MPVh. Asimismo, es necesario implementar medidas de control de infección y aislamiento en cohorte y de contacto de estos pacientes, para evitar su disemi- nación nosocomial y la infección mixta entre MPVh y VRS $^{63,64}$.

\section{Resumen}

Metapneumovirus humano (MPVh), agente de infección respiratoria aguda baja (IRAB) recientemente descrito, ha sido detectado en 5,4\% de lactantes chilenos hospitalizados por IRAB, con estudio negativo para virus respiratorio sincicial, adenovirus, parainfluenza e influenza. Puede determinar bronquiolitis o neumonía en hospitalizados, en ocasiones llega a requerir conexión a ventilación mecánica y tratamiento en una unidad de cuidados intensivos. En algunos casos se presenta como apnea, situación que es más frecuente en prematuros. Está descrita su transmisión nosocomial. Presentamos el caso de un lactante de un mes de edad, con apnea, antecedente de prematurez e infección por MPVh y una probable adquisición intrahospitalaria. Se revisan las características clínicas de la infección por este agente y se discute la asociación con apnea e infección nosocomial. El MPVh debiera ser incluido en el estudio etiológico de lactantes que presentan apnea con estudio viral convencional negativo y como agente respiratorio de infección nosocomial.

\section{Referencias}

1.- Papic Z, Rodríguez 1, Larrañaga $C$, Avendaño L. Virus respiratorios en lactantes con infecciones respiratorias altas y bajas. Rev Chil Pediatr 1992; 63: 56-61.

2.- Lagos R, Avendaño L, Levine M. Vigilancia sistemática de virus influenza, respiratorio sincicial, parainfluenza y adenovirus en niños ambulatorios con infecciones respiratorias agudas. Rev Méd Chile 1999; 127: 1063-72

3.- Van den Hoogen B G, De Jong J C, Groen J, Kuiken T, de Groot R, Fouchier R A, et al. A newly discovered human pneumovirus isolated from young children with respiratory tract disease. Nat Med 2001; 7: 719-24.

4.- Avendaño L F, Palomino M A, Larrañaga C. Surveillance for respiratory syncytial virus in infants hospitalized for acute lower respiratory respiratory infection in Chile (1989 to 2000). J Clin Microbiol 2003; 541: 4879-82.

5.- Larrañaga $C$, Vicente $M, W u$ E, Carrasco $C$, Peña A M, Oñate $C$, et al. Adenovirus en niños con infecciones respiratorias agudas bajas. Rev Chil Pediatr 1988; 59: 312-7.

6.- Vega-Briceño L, Potin M, Bertrand P,
Sánchez I. Infección respiratoria por virus influenza en niños ¿Qué aprendimos durante el año 2004? Rev Méd Chile 2005; 113: 911-8.

7.- Luchsinger V, Escobar C, Avendaño L. Detección de metapneumovirus humano en niños hospitalizados por infección respiratoria en Santiago, Chile. Rev Méd Chile 2005; 133: 1059-64.

8.- Easton A J, Domachowske J B, Rosenberg H F. Animal pneumoviruses: molecular genetics and pathogenesis. Clin Microbiol Rev 2004; 17: 390-412.

9.- Herfst S, De Graaf M, Schickli J H, Tang R S, Kaur J, Yang C F, et al Recovery of human metapneumovirus genetic lineages A and B from cloned cDNA. J Virol 2004; 78: 8264-70.

10.- Mackay I M, Bialasiewicz S, Walizzamann Z, Chidlow G R, Fegredo D C, Laingam S, et al. Use of the $\mathrm{P}$ gene to genotype human metapneumovirus identifies 4 viral subtypes. J Infect Dis 2004; 190: 1913-8.

11.- Viazov S, Ratjen F, Scheidhauer R, Fiedler M, Roggendorf M. High prevalence of human metapneumovirus infection in young children and genetics heterogenicity of the viral isolates. J Clin Microbiol 2003; 41: $3043-5$.
12.- Peret T C, Boivin G, Li Y, Couillard M, Humprey C, Osterhaus A D, et al. Characterization of human metapneumovirus isolated in North America. J Infect Dis 2002; 185: 1660-3.

13.- Esper F, Martinello R A, Boucher D, Weibel C, Ferguson D, Landry M L, et al. A 1 year experience with human metapneumovirus in children aged $<5$ years. J Infect Dis 2004; 189: 1388-96.

14.- Esper F, Boucher D, Weibel C, Martinello R A, Kahn J S. Human metapneumovirus infection in the United States: clinical manifestations associated with a newly emerging respiratory infection in children. Pediatrics 2003; 111: 1407-10.

15.- Robinson J L, Lee B E, Bastien N, Li Y. Seasonality and clinical features of human metapneumovirus infection in children in Northern Alberta. J Med Virol 2005; 76: $98-105$.

16.- Noyola D E, Alpuche-Solís A G, Herrera-Díaz A, Soria-Guerra R E, Sánchez-Alvarado J, López-Reville R. Human metapneumovirus in Mexico: epidemiological and clinics aspects characteristics. J Med Microbiol 2005; 54 : 969-74.

17.- Carar M J, McCormack G P, Crowley B. 
Human metapneumovirus associated respiratory tract infections in the Republic of Ireland during the influenza season of 2003-2004. Clin Microbiol Infect 2005; 11: 366-71.

18.- Xepapadaki P, Psarras S, Bossios A, Tsolia M, Gourgiotis D, Liapi-Adamidau G, et al. Human metapneumovirus as a causative agent of acute bronchiolitis in infants. J Clin Virol 2004; 30: 267-70.

19.- García-García M L, Calvo C, Martín F, Pérez-Breña P, Acosta B, Casas I. Human metapneumovirus infections in hospitalized infants in Spain. Arch Dis Child 2006; 91: 290-5.

20.- Sarasini A, Percivalle E, Rovida F, Campanini G, Genini E, Torsellini M, et al. Detection and pathogenicity of human metapneumovirus respiratory infection in pediatric italian patients during a winterspring season. J Clin Virol 2006; 35: 59-68.

21.- Nissen M D, Siebert D J, Mackay I M, Sloots T P, Whiters S J. Evidence of human metapneumovirus in Australian children. Med J Austr 2002; 176: 188.

22.- Keida A, Iritani N, Kubo H, Shiomi M, Kohdera V, Murakami T. Seasonal distribution and phylogenetic analysis of human metapneumovirus among children in Osaka city, Japan. J Clin Virol 2006; 35 : 394-9.

23.- Samransamruajkit R, Thanasugarn W, Prapphal N, Theamboonlers A, Poovorawan Y. Human metapneumovirus in infants and young children in Thailand with lower respiratory tract infections; molecular characteristics and clinical presentations J Infect 2006; 52: 254-63.

24. - IJpma F F, Beekhuis D, Cotton M F, Pieper C H, Kimpen J L, van den Hoogen B $\mathrm{G}$, et al. Human metapneumovirus infection in hospital referred South African children. J Med Virol 2004; 73: 486-93.

25.- Madhi S A, Ludewick H, Abed Y, Klugman K P, Boivin G. Human metapneumovirus- associated lower respiratory tract infections among human immunodeficiency virus type 1 (HIV -1) infected and HIV-1 uninfected African infants. Clin Infect Dis 2003; 37: 1705-10.

26.- Gray G C, Capuano A W, Setterquist S F, Sánchez J L, Neville J S, Olson J, et al. Human metapneumovirus, Peru. Emerg Infect Dis 2006; 12: 347-50.

27. - Galiano M, Videla C, Puch S S, Martínez A, Echavarría M, Carballal G. Evidence of human metapneumovirus in children in Argentina. J Med Virol 2004; 72: 299-303.

28.- Cuevas L E, Nasser A M, Dove W, Gurgel R Q, Grensill J, Hart C A. Human metapneumovirus and respiratory syncytial virus, Brazil. Emerg Infect Dis 2003; 9: 1626-8.

29.- Williams J C V, Wang C K, Yang C F, Tollefson S J, House F S, Heck J M, et al. The role of human metapneumovirus in upper respiratory tract infection in children. A 20 year experience. J Infect Dis 2006; 193: $387-95$

30.- Williams J V, Harris P A, Tollefson S J, Halburnt- Rush L L, Pingsterhaus J M, Edwards K, et al. Human metapneumovirus and lower respiratory tract disease in otherwise healthy infants and children. $\mathrm{N}$ Engl J Med 2004; 350. 443-50.

31.- Pelletier G, Déry P, Abed Y, Boivin G. Respiratory tract reinfections by the new human metapneumovirus in an immunocompromised child. Emerg Infect Dis 2002; 8: 976-8.

32.- Stockton J, Stephenson I, Fleming D, Zambon M. Human metapneumovirus as a cause of community acquired respiratory illness. Emerg Infect Dis 2002; 8: 897-901.

33. - Boivin G, De Serres G, Cote S, Gilca R, Abed Y, Rochette L et al. Human metapneumovirus infections in hospitalized children. Emerg Infect Dis 2003; 9: 634-40.

34.- Boivin G, Abed Y, Pelletier G, Ruel L, Moisan D, Cote S, et al. Virological features and clinical manifestations associated with human metapneumovirus; a new paramixovirus responsible for acute respiratory tract infections in all age group. J Infect Dis 2002; 186: 1330-4.

35.- Falsey A R, Erdman D, Anderson L J, Walsh E E. Human metapneumovirus infections in young and elderly infants. J Infect Dis 2003; 187: 785-90.

36. - Peiris J S, Tang W H, Chan K H, Khong P L, Guan Y, Lau Y L, et al. Children with respiratory disease associated with metapneumovirus in Hong Kong. Emerg Infect Dis 2003; 9: 628-33.

37. - van den Hoogen B, van Doornum G, Fockens J C, Cornelissen J J, Beyer W, De Groot R, et al. Prevalence and clinical symptoms of human metapneumovirus infection in hospitalized patients. J Infect Dis 2003; 188: 1571-7.

38. - Hamelin ME, Abed Y, Boivin G. Human metapneumovirus: a new player among respiratory viruses. Clin Infect Dis 2004; 38: 983-90.

39.- Williams J V, Tollefson S J, Nair S, Chonmaitree T. Association of human metapneumovirus with acute otitis media. Int J Pediatr Otorhinolaryngol 2006; 70: 1189-93.

40.- Suzuki A, Watanabe O, Okamoto M, Endo H, Yano H, Suetake M, et al. Detection of human metapneumovirus from children with acute otitis media. Pediatr
Infect Dis J. 2005; 24: 655-7.

41.- Jartti T, van den Hoogen B, Garofalo R P, Osterhaus A D, Ruuskanen O. Metapneumovirus and acute wheezing in children. Lancet 2002; 360: 1393-4.

42. - Mahalingam S, Schwarze J, Zaid A, Nissen M, Sloots T, Tauro S, et al. Perspective on the host response to human metapneumovirus infection: what can we learn from respiratory syncytial virus infections? Microbes Infect 2005; 8: 285 93.

43.- Freymouth F, Vabret A, Legrand L, Eterradosi N, Lafay- Delaire F, Brouard J, et al. Presence of the new human metapneumovirus in French children with bronchiolitis. Pediatr Infect Dis J 2003; 22: 92-4.

44.- McAdam A J, Hasenbein M E, Feldman H A, Cole S E, Offermann J T, Riley A M, et al. Human metapneumovirus in children tested at a tertiary care hospital. J Infect Dis 2004; 190: 20-6.

45.- García M L, Calvo C, Del Valle M, López M R, Casas I, Díaz-Delgado R, et al. Infecciones respiratorias por metapneumovirus en lactantes hospitalizados. An Pediatr (Barc) 2004; 61: 213-8.

46.- Alto W A. Human metapneumovirus: a newly described respiratory tract pathogen. J Am Board Fam Pract 2004, 17: 466-9.

47. - Van den Hoogen B, Osterhaus A, Foucher R. Clinical impact and diagnosis of human metapneumovirus infection. Pediatr Infect Dis J 2004; 23: 25-32.

48. - Sample M G, Cowell A, Dove W, Grensill J, McNamara P S, Halfhide C, et al. Dual infections of infants by human metapneumovirus and human respiratory syncytial virus is strongly associated with severe bronchiolitis. J Infect Dis 2005; 191: 382-6.

49.- Sumino K C, Agapov E, Pierce R A, Trulock E P, Pfeifer D, Ritter J H, et al. Detection of human metapneumovirus by real time polymerase chain reaction and histopathological assessment. J Infect Dis 2005; 192: 1052-60.

50.- Williams J V, Tollefson S J, Heymann P W, Carper H T, Patrie J, Crowe J E. Human metapneumovius infection in children hospitalized for wheezing. J Allergy Clin Immunol 2005; 115: 1311-2.

51.- Cote S, Abed Y, Boivin G. Comparative evaluation of real time PCR assays for detection of the human metapneumovirus. J Clin Microbiol 2003; 41: 3631-5.

52.- Dollner H, Risnes K, Radtke A, Nordbo S A. Outbreak of human metapneumovirus infection in Norwegian children. Pediatr Infect Dis J 2004; 23: 436-40.

53.- Schildgen O, Simon A, Wilkesmann A, 
Williams J, Kapper B, Eis-Hubinger A M, et al. The human metapneumovirus: biology, epidemiological features and clinical characteristic of infection. Rev Med Microbiol 2006; 17: 11-25.

54.- Wilkesmann A, Schildgen O, Eis-Hubinger A M, Geikowski T, Glatzel T, Lentze MJ et al. Human metapneumovirus infections cause similar symptoms and clinical severity as respiratory syncytial virus infections. Eur J Pediatr 2006; 165: 467-75.

55.- Kneyber M C, Brandenburg A H, de Groot R, Joosten K F, Rothbarth P H, Ott A, et al. Risk factors for respiratory syncytial virus associated apnea. Eur J Pediatr 1998; 157: 331-5.

56.- Anas N, Boettrich C, Hall C B, Brooks J G. The association of apnea and respiratory syncytial virus infection in infants.
J Pediatr 1982; 101: 65-80.

57.- Lindgren C, Jing L, Graham B, Grögaard J, Sundell H. Respiratory syncytial virus reinforces reflex apnea in young lambs. Pediatr Res 1992; 31: 381-5.

58.- Chano F, Rousseau C, Laferrière C, Couillard M, Charest H. Epidemiological survey of human metapneumovirus infection in a large pediatric tertiary care center. J Clin Microbiol 2005; 43: 5520-5.

59.- Morrow B, Hatherill M, Smuts H, Yeats J, Pitcher R, Argent A C. Clinical course of hospitalized children infected with human metapneumovirus and respiratory syncytial virus. J Paediatr Child Health 2006; 42: 174-8.

60.- Cane P A, van den Hoogen B, Chakrabarti S, Fegan C D, Osterhaus A. Human metapneumovirus in a haematopoietic stem cells transplant recipient with fatal lower respiratory tract disease. Bone Marrow Transpl 2003; 31: 309-10.

61.- Greensill J, McNamara P S, Dove W, Flanagan B, Smyth R L, Hart A. Human metapneumovirus in severe respiratory syncytial virus bronchiolitis. Emerg Infect Dis 2003; 9: 372-5.

62.- Schildgen O, Geikowski T, Glatzel T, Simon A, Wilkesmann A, Roggendorf M, et al. New variant of the human metapneumovirus (HMPV) associated with acute and severe exacerbations of asthma bronchiale. J Clin Virol 2004; 31: 283-8.

63.- Mejías A, Chávez- Bueno S, Ramilo O. Human metapneumovirus: a not so new virus. Pediatr Infect Dis J 2004; 23: 1-7.

64.- Crowe J E. Human metapneumovirus as a major cause of human respiratory tract disease. Pediatr Infect Dis J 2004; 23: S215-21. 